Internat. J. Math. \& Math. Sci.

Vol. 24, No. 3 (2000) 187-192

S0161171200010061

(c) Hindawi Publishing Corp.

\title{
HOMOCLINIC ORBITS AND LIE ROTATED VECTOR FIELDS
}

\author{
JIE WANG and CHEN CHEN
}

(Received 2 April 1999)

\begin{abstract}
Based on the definition of Lie rotated vector fields in the plane, this paper gives the property of homoclinic orbit as parameter is changed and the singular points are fixed on Lie rotated vector fields. It gives the conditions of yielding limit cycles as well.
\end{abstract}

Keywords and phrases. Lie rotated vector fields, Lie bracket, one parameter group, homoclinic orbits.

2000 Mathematics Subject Classification. Primary 34C05.

1. Introduction. The rotated vector fields have been considered as a very important tool which is efficient in the study of the numbers of limit cycles and the distribution of homoclinic orbits. In this paper, we continue with the investigation of the Lie rotated vector fields [6]. Based on the definition of Lie rotated vector fields [6], we give the property of homoclinic orbit as parameter is changed and the singular points are fixed on Lie rotated vector fields. As application of Lie rotated vector fields, we prove that the definition of Lie rotated vector has nothing to do with the change of coordinate. Afterwards, we require the singular points of $X(\mu)$ not to be moved as parameter $\mu$ is changed. We study the motion of homoclinic orbits that pass through saddle points on Lie rotated vector fields and the change of a family of periodic orbits that are in the inner neighborhood of homoclinic orbit. Of course, we give some examples to illustrate the concept and notion of Lie rotated vector fields.

2. Topological properties of Lie rotated vector fields. Let $X(\mu)$ be a Lie rotated vector field. In this section, we require the singular points of $X(\mu)$ not to be moved as parameter $\mu$ is changed, i.e., the singular points are kept immovable.

THEOREM 2.1. The Lie rotated vector field is a Lie rotated vector field under the $C^{2}$ differential topological transform.

Proof. Let $\phi$ be a $C^{2}$ differential topological transform on $\mathbb{R}^{2}$, and let that $\phi \in$ $C^{2}\left(\mathbb{R}^{2}, \mathbb{R}^{2}\right), \phi(x): x \mapsto y=\phi(x), x=\left(x_{1}, x_{2}\right), y=\left(y_{1}, y_{2}\right) \in \mathbb{R}^{2}$. Let $X(\mu)$ be a Lie rotated vector field, then $Y$ is a corresponding vector field which satisfies formula

$$
L(0) \stackrel{\text { def }}{=} X(0) \wedge\left\{X_{\mu}^{\prime}(0)+[X(0), Y]\right\}>0(<0),
$$

where $X_{\mu}^{\prime}(0)$ is the derivative of the vector field $X(\mu)$ at $\mu=0$. Under the transform $\phi, X(\mu)=X(x, \mu)$ becomes

$$
\bar{X}(\mu) \stackrel{\text { def }}{=}\left(\phi_{*} X\right)(y, \mu)=D \phi\left(\phi^{-1}(y)\right) \cdot X\left(\phi^{-1}(y), \mu\right)
$$


use the same method, under the transform $\phi, Y$ becomes

$$
\bar{Y} \stackrel{\text { def }}{=}\left(\phi_{*} Y\right)(y)=D \phi\left(\phi^{-1}(y)\right) \cdot X\left(\phi^{-1}(y)\right) .
$$

For $\bar{X}(\mu)$ and the corresponding $\bar{Y}$, and $\forall y=\phi(x) \in \mathbb{R}^{2}$, we only need to prove the condition to set up

$$
\overline{L(0)}=\bar{X}(0) \wedge\left\{\bar{X}_{\mu}^{\prime}(0)+\overline{[\bar{X}(0), \bar{Y}]}\right\}>0(<0),
$$

where $\overline{[,]}$ is Lie bracket, which expresses the action to the variable $y$. Since

$$
\begin{gathered}
\bar{X}(0) \wedge \overline{[\bar{X}(0), \bar{Y}]}=D \phi(x) \cdot X(x, 0) \wedge[X(x, 0), Y(x)], \\
\bar{X}(0) \wedge \bar{X}_{\mu}^{\prime}(0)=D \phi(x) \cdot X(x, 0) \wedge X_{\mu}^{\prime}(x, 0),
\end{gathered}
$$

where $x=\phi^{-1}(y)$. From formulae (2.5) and (2.6), we can find that

$$
\bar{L}(0)=D \phi(x) \cdot L(0)
$$

where $x=\phi^{-1}(y)$. But $\phi$ is the $C^{2}$ differential topological transform, $D \phi(x) \neq 0$, and $L(0)>0($ or $<0)$, it follows that $\bar{L}(0)>0($ or $<0)$.

Theorem 2.1 illustrates that Lie rotated vector fields have nothing to do with the choice of coordinates, and the symbol of $\bar{L}(0)$ in formula (2.4) is decided by the symbol of $L(0)$ and the formula of Jacobi on the transform $\phi$.

THEOREM 2.2. Let $X(\mu)$ be a Lie rotated vector field, $\left.X(0)\right|_{p_{0}}=0$.

(1) If the index $J_{0}\left(p_{0}\right)$ of singular point $p_{0}$ of $X(0)$ is not equals to zero, then $\left.Y\right|_{p_{0}}=0$.

(2) If the index $J_{0}\left(p_{0}\right)$ of singular point $p_{0}$ of $X(0)$ is changed as $\mu$ is changed, then $\left.Y\right|_{p_{0}} \neq 0$.

Proof. (1) Using reduction to absurdity, we let $\left.Y\right|_{p_{0}} \neq 0$. From [6, Lemma 2.1], we know that $\left.\psi_{*}^{\mu} X(\mu)\right|_{\psi^{\mu}\left(p_{0}\right)}=0(\mu \neq 0)$, the proof is similar to [6, part (2) of Theorem 3.2]. For $\forall \varepsilon>0$, such that $\psi^{\mu}\left(p_{0}\right) \notin \overline{S_{\varepsilon}\left(p_{0}\right)},|\mu|<\bar{\delta}<\delta, \bar{\delta}$ is given by [6, Theorem 3.2]. Since $\left.\psi_{*}^{\mu} X(\mu)\right|_{p_{0}} \neq 0$, it is noted that $J_{\mu_{*}}\left(p_{0}\right)=0(\mu \neq 0)$ to $\partial S_{\varepsilon}\left(p_{0}\right)$ about $\psi_{*}^{\mu} X(\mu)$, but $J_{0}\left(p_{0}\right) \neq 0$ to $\partial S_{\varepsilon}\left(p_{0}\right)$ about $X(0)$. From [6, Lemma 2.3], we know that this is a contradiction, thus $\left.Y\right|_{p_{0}}=0$.

(2) The proof is similar to (1). In fact, we might as well let $\left.Y\right|_{p_{0}}=0$, then $\left.\psi_{*}^{\mu} X(\mu)\right|_{p_{0}}=$ 0 . Let $J_{0}\left(p_{0}\right)=r_{0}$ about $X(0)$, but $J_{\mu}\left(p_{0}\right)=r_{\mu}\left(r_{\mu} \neq r_{0}, \mu \neq 0\right)$ about $X(\mu)$. From [6, Lemma 2.2], we know that $J_{*_{\mu}}\left(p_{0}\right)=r_{\mu}$. Again from [6, Lemma 2.3], when $|\mu|<\bar{\delta}<\delta$, $J_{0}\left(p_{0}\right)=J_{* \mu}\left(p_{0}\right)=r_{0}$, i.e., $r_{0}=r_{\mu}$, and by the supposition $r_{0} \neq r_{\mu}$, this is a contradiction, thus $\left.Y\right|_{p_{0}} \neq 0$.

COROLLARY 2.3. Let $X(\mu)$ be a Lie rotated vector field, if $p_{01}, p_{02}, \ldots, p_{0 m}$ are $m$ elementary singular points of $X(0)$, then the corresponding vector field $Y$, certainly set up

$$
\left.Y\right|_{p_{0 j}}=0 \quad(1 \leq j \leq m) .
$$


3. The motion of homoclinic orbit. From the theory of structural stability on twodimensional mainfolds, we know that the systems which have homoclinic orbit passing through the saddle points are structurally unstable, this orbit which links saddle points can extremely be burst under trouble, thus can change the topological structure of the orbit. When $X(\mu)$ is Lie rotated vector field, and if $\mu=0, X(0)$ has homoclinic orbit passing through saddle points, we consider the change of topological structure of the orbit of $X(\mu)$ when $\mu \neq 0$.

THEOREM 3.1. Let $X(\mu)$ be Lie rotated vector field, $\Gamma_{0}=\{q(t), t \in \mathbb{R}\} \bigcup p_{0}$ is homoclinic orbit passing through the hyperbolic saddle point $p_{0}$ on limited region. If $\sigma_{0}=\left.\operatorname{div} X(0)\right|_{p_{0}} \neq 0$, then when $\mu$ is changed toward suitable direction, $\Gamma_{0}$ disappears, but it will produce unique limit cycle of $X(\mu)$ in the neighborhood of $\Gamma_{0}$, and the limit cycle is stable or unstable; but when $\mu$ is changed towards other direction, $\Gamma_{0}$ disappears, and it will not produce any limit cycle of $X(\mu)$ in the neighborhood of $\Gamma_{0}$.

Proof. We might as well let $\Gamma_{0}$ positively oriented, $\sigma_{0}<0$. From [5, Theorem 1], we know that $\Gamma_{0}$ is inner stable. Since $p_{0}$ is a hyperbolic saddle point of $X(0)$, from Corollary 2.3, we know that it certainly has $\left.Y\right|_{p_{0}}=0$, thus it has $\left.\psi_{*}^{\mu} X(\mu)\right|_{p_{0}}=0(\mu \neq 0)$. $\psi_{*}^{\mu} X(\mu)$ has homoclinic orbit $\Gamma_{0}$ passing through a hyperbolic saddle point $p_{0}$ when $\mu=0$. By [6, Lemma 2.3], when $0<|\mu|<\delta, \Gamma_{0}$ is burst in $\psi_{*}^{\mu} X(\mu)(\mu \neq 0)$, yet from the topological equivalence of orbital structure of $X(\mu)$ and orbital structure of $\psi_{*}^{\mu} X(\mu)$, we know that $\Gamma_{0}$ of $X(\mu)$ is burst when $\mu \neq 0$, i.e., when $0<\mu<\delta$, the homoclinic orbit $\Gamma_{0}$ passing through hyperbolic saddle point $p_{0}$ is burst into stable manifold $S_{\mu}$ and unstable manifold $U_{\mu}$. Since $\Gamma_{0}$ is inner stable, by continuous dependence of solution to the parameter $\mu$ and using the Poincaré-Bendixson annular regional theorem, we prove that there is a limit cycle in the neighborhood of $\Gamma_{0}$ when $\mu>0$, yet by $\sigma_{0}<0$, we know that there is only a unique stable limit cycle in the neighborhood of $\Gamma_{0}$. But when $\mu<0,|\mu|<\delta$, let there be a limit cycle in the neighborhood of $\Gamma_{0}$, this is the same discussion as above, we know that it is sure to have semi-stable limit cycle or unstable limit cycle, this is contradiction with $\sigma_{0}<0$.

Using Theorems 2.2 and 3.1, we can easily prove the following corollary.

COROLlaRY 3.2. Let $X(\mu)$ be a Lie rotated vector field, $\Gamma_{0}=\{q(t), t \in \mathbb{R}\} \bigcup p_{0}$ is homoclinic orbit passing through the saddle point $p_{0}$ of $X(0)$ on the limited region, $\sigma_{0}=\left.\operatorname{div} X(0)\right|_{p_{0}} \neq 0$. If $J_{0}\left(p_{0}\right) \neq 0$ (or $J_{0}\left(p_{0}\right)=0$, but $\left.\left.Y\right|_{p_{0}}=0\right)$ ), then when $\mu$ is changed towards a suitable direction, $\Gamma_{0}$ disappears, but it will produce a unique limit cycle of $X(\mu)$ in the neighborhood of $\Gamma_{0}$, and the limit cycle is stable or unstable; but when $\mu$ is changed towards the other direction, $\Gamma_{0}$ disappears, and it will not produce any limit cycle of $X(\mu)$ in the neighborhood of $\Gamma_{0}$.

Using [1, Theorems 45 and 49], [4, Theorem 1.2], and Theorem 2.2 of this paper, we have the following corollary.

COROLLARY 3.3. Let $X(\mu)$ be a Lie rotated vector field, $\Gamma_{0}=\{q(t), y \in \mathbb{R}\} \cup p_{0}$ is homoclinic orbit passing through saddle point $p_{0}$ of $X(0)$ on the limited region, $\sigma_{\mu}=$ $\left.\operatorname{div} X(\mu)\right|_{p_{0}}=0(|\mu| \ll 1$ and $|\mu|<\delta), I_{0}=\int_{\Gamma_{0}} \sigma_{0} d t \neq 0$. If $J_{0}\left(p_{0}\right) \neq 0$ (or $J_{0}\left(p_{0}\right)=0$, 
but $\left.Y\right|_{p_{0}}=0$ ), then when $\mu$ is changed towards a suitable direction, $\Gamma_{0}$ disappears, but it will produce a unique limit cycle of $X(\mu)$ in the neighborhood of $\Gamma_{0}$, and the limit cycle is stable or unstable; but when $\mu$ is changed towards other direction, $\Gamma_{0}$ disappears, and it will not produce any limit cycle of $X(\mu)$ in the neighborhood of $\Gamma_{0}$.

EXAMPLE 3.4. Let $X(\mu)=\left(2 x_{2}, 2 x_{1}-3 x_{1}^{3}-x_{2}\left(x_{1}^{3}-x_{1}^{2}+x_{2}^{2}\right)+\mu x_{2}^{3}\right)$, when $\mu=0$, $p_{0}=(0,0), p_{1}=(2 / 3,0)$ are elementary singular points of $X(0)$, where $p_{0}$ is saddle point, $p_{1}$ is an unstable focal point. Since $\Gamma_{0}: x_{1}^{3}-x_{1}^{2}+x_{2}^{2}=0$ is homoclinic orbit passing through the hyperbolic saddle point $p_{0}$ of $X(0)$ (this example is shown in [5]). By Corollary 2.3, we can take $Y=\left(0, \beta\left(2 x_{1}-3 x_{1}^{2}\right)\right)$, where $\beta \in \mathbb{R}$. For, $\forall \varepsilon>0$, $\varepsilon$ is taken small enough, we make open neighborhoods $S_{\varepsilon}\left(p_{0}\right)$ and $S_{\varepsilon}\left(p_{1}\right)$ of $p_{0}$ and $p_{1}$, respectively, then there is a limited region $D \subset \mathbb{R}^{2}, \Gamma_{0} \subset D$, order $\beta=\varepsilon^{3}>0$, at the ordinary point of $X(0)$ of $D \backslash\left\{S_{\varepsilon}\left(p_{0}\right) \cup S_{\varepsilon}\left(p_{1}\right)\right\}$, we have

$$
L(0)=2 \beta\left(2 x_{1}-3 x_{1}^{2}+x_{2}^{3}\right)^{2}+2 \beta\left(4 x_{2}^{2}\left(1-3 x_{1}\right)-x_{2}^{6}\right)+2 x_{2}^{4}>0,
$$

i.e., $X(\mu)$ constitutes Lie rotated vector field on $D$. Take $|\mu| \ll 1$ and $|\mu|<\delta$, note $\sigma_{\mu}=$ $\left.\operatorname{div} X(\mu)\right|_{p_{0}}=0$, again from [5], we can know that $I_{0}=\int_{\Gamma_{0}} \sigma_{0} d t<0$. By Corollary 3.3, when $\mu<0, \Gamma_{0}$ disappears, but it will produce a unique stable limit cycle of $X(\mu)$ in the neighborhood of $\Gamma_{0}$; but when $\mu>0, \Gamma_{0}$ disappears, and it will not produce any limit cycle in the neighborhood of $\Gamma_{0}$.

Theorem 3.1 and Corollary 3.2 require $\sigma_{0} \neq 0$, Corollary 3.3 requires $\sigma_{\mu}=0(0 \leq$ $|\mu|<\delta$ and $|\mu| \ll 1$ ), using [3, Lemmas 8 and 9] and the proven method of Theorem 3.1 in this paper, we have the following corollary.

COROLLARY 3.5. Let $X(\mu)$ be a Lie rotated vector field, $\Gamma_{0}=\{q(t), t \in \mathbb{R}\} \bigcup p_{0}$ is homoclinic orbit passing through the saddle point $p_{0}$ of $X(0)$ on the limited region, $\Gamma_{0}$ is stable (unstable). If $J_{0}\left(p_{0}\right) \neq 0$ (or $J_{0}\left(p_{0}\right)=0$, but $\left.Y\right|_{p_{0}}=0$ ), then when $\mu$ is changed towards a suitable direction (towards the other direction), $\Gamma_{0}$ disappears, but it at least produces a limit cycle in the neighborhood of $\Gamma_{0}$, the limit cycle is stable (unstable); when $\mu$ is changed towards the other direction (towards a suitable direction), $\Gamma_{0}$ disappears, but it will not produce any limit cycle in the neighborhood of $\Gamma_{0}$.

EXAMPLE 3.6. Let $X(\mu)=\left(2 x_{2}, 2 x_{1}-3 x_{1}^{2}-x_{2}\left(x_{1}^{3}-x_{1}^{2}+x_{2}^{2}\right)+\mu x_{2}\right)$, when $\mu=0$, the state of $X(0)$ is same as Example 3.4, we yet take $Y=\left(0, \beta\left(2 x_{1}-3 x_{1}^{2}\right)\right)$. For, $\forall \varepsilon>0$, $\varepsilon$ is sufficiently small, order $\beta=\varepsilon>0$, then there is a region $D \subset \mathbb{R}^{2}, \Gamma_{0} \subset D$, at the ordinary point of $X(0)$, we have

$$
L(0)=2 \beta\left(2 x_{1}-3 x_{1}^{2}+x_{2}^{3}\right)^{2}+2 \beta\left(4 x_{2}^{2}\left(1-3 x_{1}\right)-x_{2}^{6}\right)+2 x_{2}^{2}>0,
$$

i.e., $X(\mu)$ constitutes a Lie rotated vector field on $D$. Take $|\mu| \ll 1,|\mu|<\delta$, by $\sigma_{0}=$ $\left.\operatorname{div} X(0)\right|_{p_{0}}=0, \sigma_{\mu}=\left.\operatorname{div} X(\mu)\right|_{p_{0}}=\mu(\mu \neq 0)$, and $I_{0}=\int_{\Gamma_{0}} \sigma_{0} d t<0$, we know that $\Gamma_{0}$ is inner stable, $J_{0}\left(p_{0}\right)=-1 \neq 0$. From Corollary 3.5, when $\mu<0$, in the neighborhood of $\Gamma_{0}$, at least produces a limit cycle; when $\mu>0$, in the neighborhood of $\Gamma_{0}$, it does not produce any limit cycle.

Now we consider that $X(\mu)$ is a Lie rotated vector field. If $\mu=0, \Gamma_{0}$ is homoclinic orbit passing through saddle point $p_{0}$ of $X(0)$ on the limited region, and the inner neighborhood of $\Gamma_{0}$ is imbued a family of periodic orbits, where $\sigma_{0}=I_{0}=0$. 
LEMмA 3.7. Let $X$ be a $C^{1}$ vector field, the limited region $\Delta_{1} \subset \mathbb{R}^{2}$ is imbued a family of periodic orbits $L_{h}$ for $X, h \in(a, b) \subset \mathbb{R}$, then for all $C^{1}$ vector fields $Y$, we have

$$
A_{0}(h)=\int_{L_{h}}(X \wedge[X, Y]) \cdot \exp \left\{-\int_{0}^{t} \operatorname{div} X d t\right\} d t=0,
$$

where $h \in(a, b)$.

Proof. Using the formula of Theorem 2.3 of Chapter 3 in $\S 2$ of [2], we have

$$
X \wedge[X, Y]=\langle X, \nabla(X \wedge Y)\rangle-(X \wedge Y) \cdot \operatorname{div} X .
$$

Both sides of (3.4) are multiplied by the factor $\exp \left\{-\int_{0}^{t} \operatorname{div} X d t\right\}$, and both sides are integrated along the circuit of $L_{h}$, let the period of $L_{h}$ be $\omega(h)$, using [7, formula (2.16)], we have

$$
\begin{aligned}
\int_{L_{h}} & (X \wedge[X, Y]) \cdot \exp \left\{-\int_{0}^{t} \operatorname{div} X d t\right\} d t \\
= & \int_{L_{h}}\langle X, \nabla(X, Y)\rangle \cdot \exp \left\{-\int_{0}^{t} \operatorname{div} X d t\right\} d t+\int_{L_{h}}(X \wedge Y) \cdot \frac{d}{d t}\left\{\exp \left(-\int_{0}^{t} \operatorname{div} X d t\right)\right\} d t \\
= & \int_{L_{h}}\langle X, \nabla(X, Y)\rangle \cdot \exp \left\{-\int_{0}^{t} \operatorname{div} X d t\right\} d t-\int_{L_{h}} \frac{d}{d t}(X \wedge Y) \cdot \exp \left\{-\int_{0}^{t} \operatorname{div} X d t\right\} d t \\
& +\left[(X \wedge Y) \cdot \exp \left\{-\int_{0}^{t} \operatorname{div} X d t\right\}\right]_{0}^{\omega(h)}=0 .
\end{aligned}
$$

This proof is completed.

THEOREM 3.8. Let $X(\mu)$ be a Lie rotated vector field, $\Gamma_{0}=\{q(t), t \in \mathbb{R}\} \bigcup p_{0}$ is a homoclinic orbit passing through the saddle point $p_{0}$ of $X(0)$ on limited region. If the inner neighborhood $\Delta \subset \mathbb{R}^{2}$ of $\Gamma_{0}$ is imbued a family of periodic orbits $L_{h}, h \in(a, b) \subset \mathbb{R}$, then when $\mu \neq 0$, the inner neighborhood $\Delta$ of $\Gamma_{0}$ will not produce any closed orbit of $X(\mu)$.

Proof. By [7, Theorem 4.10], when $\mu \neq 0$, on the undisappeared $L_{h_{0}}$, we have

$$
A_{1}\left(h_{0}\right)=\int_{L_{h_{0}}}\left(X(0) \wedge X_{\mu}^{\prime}(0)\right) \cdot \exp \left\{-\int_{0}^{t} \operatorname{div} X(0) d t\right\} d t=0,
$$

where $h_{0} \in(a, b)$. But from Lemma 3.7, we have

$$
A_{1}\left(h_{0}\right)=A_{1}\left(h_{0}\right)+A_{0}\left(h_{0}\right)=\int_{L_{h_{0}}} L(0) \cdot \exp \left\{-\int_{0}^{t} \operatorname{div} X(0) d t\right\} d t \neq 0 .
$$

It follows that the proof is completed.

EXAMPLE 3.9. Let $X(\mu)=\left(2 x_{2}, 2 x_{1}-3 x_{1}^{2}+\mu x_{2}\right)$, when $\mu=0, p_{0}=(0,0)$ is hyperbolic saddle point of $X(0), p_{1}=(2 / 3,0)$ is the center of $X(0), \Gamma_{0}: x_{1}^{3}-x_{1}^{2}+x_{2}^{2}=0$ is homoclinic orbit passing through $p_{0}$ of $X(0)$, yet take $Y=\left(0, \kappa\left(2 x_{1}-3 x_{1}^{2}\right)\right)$. When $\kappa$ 
is taken arbitrary sufficient small, $0<\kappa \ll 1$, then there is region $D \subset \mathbb{R}^{2}, \Gamma_{0} \subset D$, at all ordinary points of $X(0)$ on $D$, we have

$$
L(0)=2 x_{2}^{2}\left(1+4 \kappa\left(1-3 x_{1}\right)\right)+2 \kappa\left(2 x_{1}-3 x_{1}^{2}\right)^{2}>0,
$$

i.e., $X(\mu)$ constitutes a Lie rotated vector field on $D$. From Theorem 3.8, we can know that there is not any closed orbit in the inner neighborhood of $\Gamma_{0}$ when $\mu \neq 0$.

Acknowledgement. The authors express grateful thanks to Professor Yaoxian Wang for his help and direction in the work.

\section{REFERENCES}

[1] A. A. Andronov, E. A. Leontovich, I. I. Gordon, and A. G. Maīer, Theory of Bifurcations of Dynamic Systems on a Plane, Halsted Press [A division of John Wiley \& Sons], New York-Toronto, Ont., 1973. MR 49\#9345.

[2] S. S. Chen and W. H. Chen, Lectures in Differential Geometry, Beijing Univ. Press, Beijing, 1983.

[3] B. Y. Feng and M. Qian, On the stability of a saddle-point separatrix loop and an analytical criterion for its bifurcation limit cycles, Acta Math. Sin. 28 (1985), no. 1, 53-70. Zbl 583.34020.

[4] D. J. Luo and D. M. Zhu, The stability of homoclinic loop and the uniqueness for generating limit cycles, Chin. Ann. Math. Ser. A 11 (1990), no. 1, 95-103. Zbl 705.34024.

[5] Z. E. Ma and E. N. Wang, The stability of a loop formed the separatrix of saddle point and the condition to produce a limit cycle, Chinese Ann. Math. Ser. A. 4 (1983), no. 1, 105-110.

[6] J. Wang and C. Chen, Singular points and Lie rotated vector fields, Int. J. Math. Math. Sci. 24 (2000), no. 3, 185-191.

[7] Y. Q. Ye, S. L. Cai, L. S. Chen, K. C. Huang, D. J. Luo, Z. E. Ma, E. N. Wang, M. S. Wang, and X. A. Yang, Theory of Limit Cycles, Translations of Mathematical Monographs, vol. 66, American Mathematical Society, Providence, R.I., 1986, Translated from the Chinese by Chi Y. LO. MR 88e:58080. Zbl 588.34022.

Jie WANG ANd Chen Chen: School of Electric Power, Shanghai JiAotong University, SHANGHAI, 200030, CHINA 


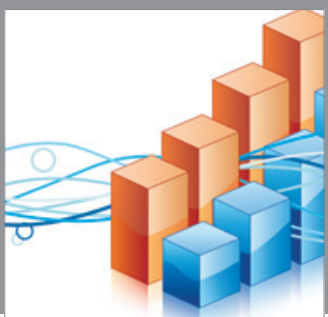

Advances in

Operations Research

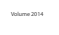

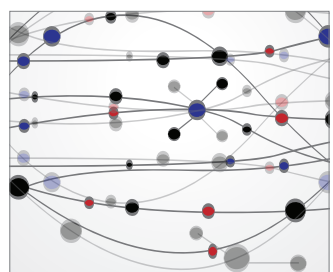

\section{The Scientific} World Journal
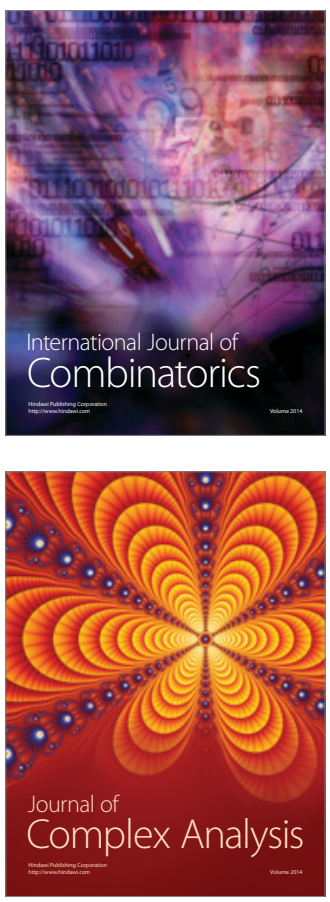

International Journal of

Mathematics and

Mathematical

Sciences
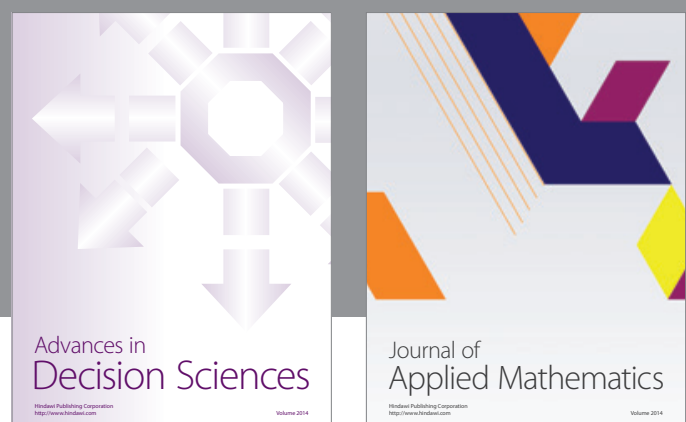

Journal of

Applied Mathematics
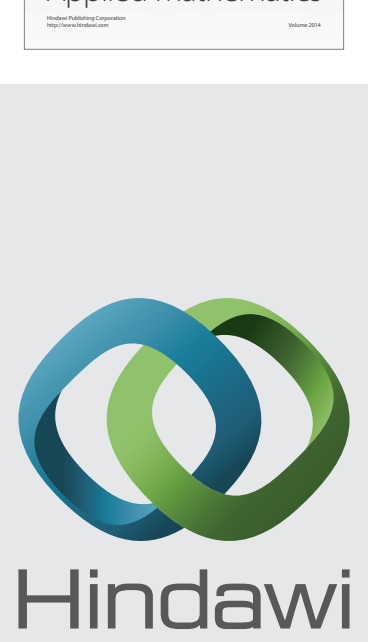

Submit your manuscripts at http://www.hindawi.com
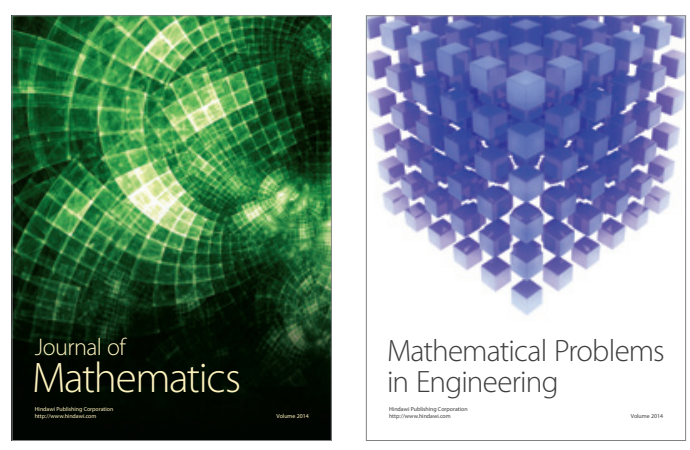

Mathematical Problems in Engineering
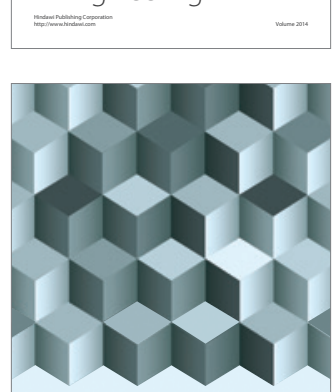

Journal of

Function Spaces
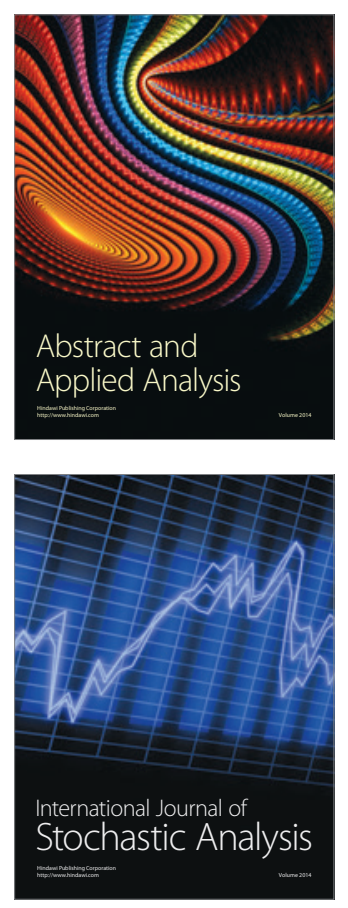

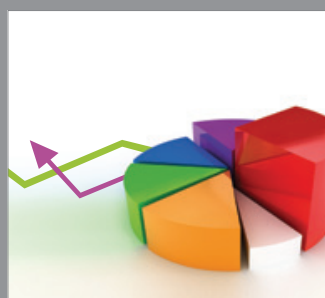

ournal of

Probability and Statistics

Promensencen
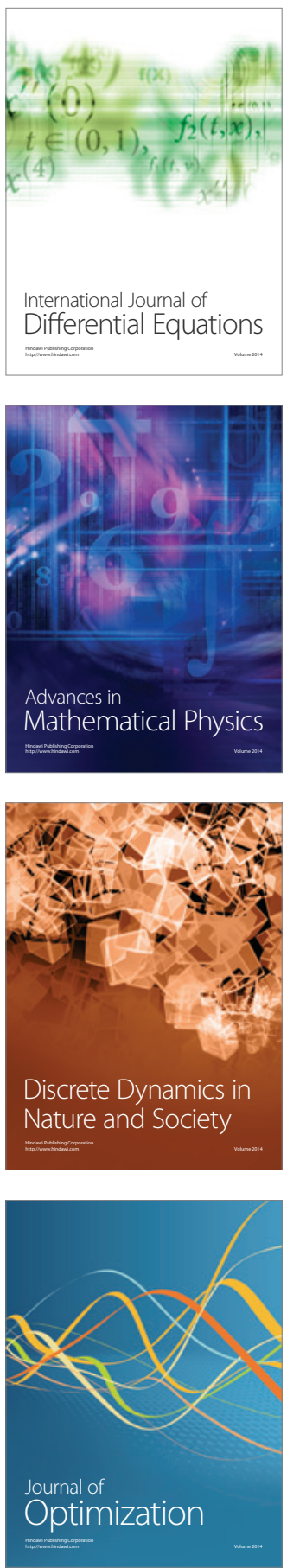